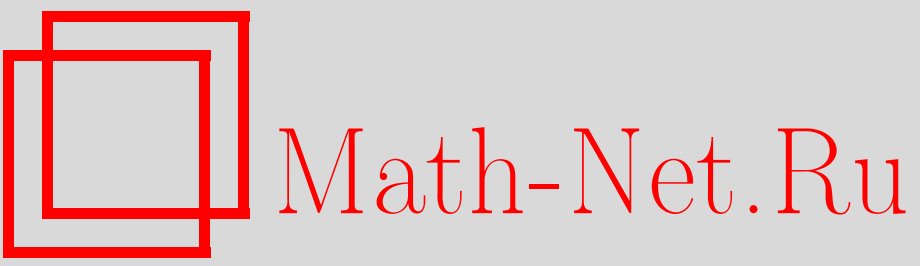

Ю. Н. Орлов, К выводу уравнений гидродинамики в рамках функциональной гипотезы Боголюбова, ТМФ, 2003, том 134, номер 3, 501-512

DOI: https://doi.org/10.4213/tmf167

Использование Общероссийского математического портала Math-Net.Ru подразумевает, что вы прочитали и согласны с пользовательским соглашением

http://www.mathnet.ru/rus/agreement

Параметры загрузки:

IP: 54.237 .206 .68

26 апреля 2023 г., 14:00:11 
ТЕОРЕТИЧЕСКАЯ

И МАТЕМАТИЧЕСКАЯ

ФИЗИКА

Том 134, № 3

март, 2003

(C) 2003 г.

\section{К ВЫВОДУ УРАВНЕНИЙ ГИДРОДИНАМИКИ В РАМКАХ ФУНКЦИОНАЛЬНОЙ ГИПОТЕЗЫ БОГОЛЮБОВА}

Функциональная гипотеза Боголюбова обобщена на случай многочастичного взаимодействия, зависящего как от координат, так и от импульсов частиц. В качестве примера рассматриваются две слаборелятивистские модели: модель Дарвина в теории заряженных частиц и модель Фока в общей теории относительности. Для них на основе цепочки уравнений ББГКИ вычисляются слаборелятивистские поправки к классическим коэффициентам переноса, а также определяются условия, при которых отсутствует взаимно однозначная связь между параметрами локально-равновесного распределения и гидродинамическими величинами.

Ключевые слова: цепочка Боголюбова, слаборелятивистское приближение, уравнения гидродинамики.

\section{1. ФУНКЦИОНАЛЬНАЯ ГИПОТЕЗА}

Одной из центральных задач статистической механики является нахождение коэффициентов переноса, связывающих (в линейной теории) термодинамические потоки с градиентами параметров, характеризуюших конкретную систему (обычно это масса, полный импульс и энергия). Эти связи, будучи подставленными в законы сохранения, приводят к уравнениям вязкой гидродинамики и теплопроводности. Исходным пунктом при выводе законов сохранения являются динамические уравнения (в гамильтоновой или лагранжевой формах) и основанное на них уравнение Лиувилля для функции распределения частиц системы в фазовом пространстве. Вывод уравнений гидродинамики из уравнения Лиувилля был дан Боголюбовым в работах [1], [2].

Ниже исследуются особенности вывода уравнений гидродинамики для систем с вырожденными связями меж ду гидродинамическими переменными и параметрами локально-равновесного распределения. В качестве примера рассмотрена слаборелятивистская функция Гамильтона, моделируюшая системы с запаздыванием взаимодействия [3], [4], приводятся выражения для необратимых потоков и вычисляются слаборелятивистские поправки к классическим коэффициентам переноса на основе слаборелятивистской цепочки уравнений Боголюбова-Борна-Грина-Кирквуда-Ивона (ББГКИ).

\footnotetext{
${ }^{*}$ Институт прикладной математики им. М. В. Келдыша РАН, Москва, Россия
} 
Приведем краткое изложение функциональной гипотезы Боголюбова [2], обобщенной на случай систем с многократным взаимодействием, зависящим от координат и импульсов частиц в декартовом фазовом пространстве. Рассматривается система из $N$ тождественных частиц без внутренней структуры, заключенных в объеме $\Omega$. Гамильтониан системы представляется в виде суммы величин унарного, бинарного и т.д. типов, симметричных относительно перестановки индексов, нумерующих частицы,

$$
H_{N}=\sum_{k=1}^{K} \sum_{1 \leqslant i_{1}<\cdots<i_{k} \leqslant N} h_{k}\left(\mathbf{x}_{i_{1}}, \ldots, \mathbf{x}_{i_{k}}\right) .
$$

Здесь $\mathbf{x}_{i}=\left(\mathbf{q}_{i}, \mathbf{p}_{i}\right)$ - фазовая координата $i$-й частицы, $\mathbf{q}_{i}, \mathbf{p}_{i} \in \mathbb{R}^{n}, n$ - размерность координатного пространства. Такое усложнение вида гамильтониана по сравнению со стандартным случаем учета только парных (бинарных) взаимодействий оправданно, так как известны модели с тернарным взаимодействием, например гамильтониан Фока [5] в слаборелятивистском приближении общей теории относительности.

Далее вводится функция распределения $(\Phi \mathrm{P})$ всей системы $F_{N}(x, t)$ как плотность вероятности нахождения системы в окрестности фазовой точки $x=\left(\mathrm{x}_{1}, \ldots, \mathbf{x}_{N}\right)$ в момент времени $t$. Ее эволюция определяется уравнением Лиувилля

$$
\partial_{t} F_{N}(x, t)=\left[H_{N}, F_{N}\right]
$$

где $[\cdot, \cdot]$ - классическая скобка Пуассона. Вводятся также $s$-частичные $Ф$ Р комплексов частиц и условие нормировки

$$
\begin{gathered}
F_{s}\left(x^{s}, t\right)=\Omega^{s} \int d \mathbf{x}_{s+1} \ldots d \mathbf{x}_{N} F_{N}, \quad x^{s}=\left(\mathbf{x}_{1}, \ldots, \mathbf{x}_{s}\right), \\
\lim _{\Omega \rightarrow \infty} \frac{1}{\Omega} \int d \mathbf{x}_{1} F_{1}\left(\mathbf{x}_{1}, t\right)=1, \quad F_{s}=\lim _{\Omega \rightarrow \infty} \frac{1}{\Omega} \int d \mathbf{x}_{s+1} F_{s+1} .
\end{gathered}
$$

В термодинамическом пределе $(N \rightarrow \infty, \Omega \rightarrow \infty, N / \Omega \rightarrow 1 / \omega)$ из соотношений $(1.2)$, (1.3) следуют уравнения эволюции $s$-частичных $\Phi$, называемые цепочкой уравнений ББГКИ,

$$
\begin{aligned}
& \partial_{t} F_{s}\left(x^{s}, t\right)=\left[H_{s}, F_{s}\right]+\sum_{k=2}^{K} \sum_{m=1}^{k-1} \sum_{1 \leqslant i_{1}<\cdots<i_{k-1} \leqslant s} \frac{1}{m ! \omega^{m}} \times \\
& \quad \times \int d \mathbf{x}_{s+1} \ldots d \mathbf{x}_{s+m}\left[h_{k}\left(\mathbf{x}_{i_{1}}, \ldots, \mathbf{x}_{i_{k-l}}, \mathbf{x}_{s+1}, \ldots, \mathbf{x}_{s+m}\right), F_{s+m}\left(x^{s+m}, t\right)\right] .
\end{aligned}
$$

Если слагаемые в гамильтониане (1.1) инвариантны относительно сдвига по координатам, то тем же свойством обладают и стационарные решения цепочки (1.4), которые будем обозначать $F_{s}^{0}$. В частности, функция $F_{1}^{0}$ однородна по $\left|\mathbf{q}_{1}\right|, F_{2}^{0}$ зависит только от модуля разности $\left|\mathbf{q}_{12}\right|=\left|\mathbf{q}_{1}-\mathbf{q}_{2}\right|$ и т.д. Вблизи равновесного состояния ФР модифицируются: вводятся параметр неоднородности $\mu$, имеющий порядок отношения радиуса действия межмолекулярных сил к характерному размеру макроскопической неоднородности, и дополнительная модифицированная координата $\mathbf{r}=\mu \mathbf{q}_{1}$. Значение $\mu=0$ 
отвечает пространственно однородному состоянию. ФР переопределяются следующим образом:

$$
F_{1}\left(x_{1}, t\right) \mapsto \widetilde{F}_{1}\left(\mathbf{r}, \mathbf{p}_{1}, t ; \mu\right) ; \quad F_{s}\left(x^{s}, t\right) \mapsto \widetilde{F}_{s}\left(\mathbf{r}, x^{s}, t ; \mu\right), \quad s \geqslant 2
$$

Далее для краткости знак тильды опускаем. Вместо цепочки (1.4) получаем модифицированную цепочку

$$
\begin{aligned}
\partial_{t} F_{s}+ & \mu \frac{\partial H_{s}}{\partial p_{1}^{\alpha}} \frac{\partial F_{s}}{\partial r^{\alpha}}=\left[H_{s}, F_{s}\right]+\sum_{k=2}^{K} \sum_{m=1}^{k-1} \sum_{1 \leqslant i_{1}<\cdots<i_{k-l} \leqslant s} \frac{1}{m ! \omega^{m}} \int d \mathbf{x}_{s+1} \ldots d \mathbf{x}_{s+m} \times \\
& \times\left\{\left[h_{k}\left(\mathbf{x}_{i_{1}}, \ldots, \mathbf{x}_{i_{k-l}}, \mathbf{x}_{s+1}, \ldots, \mathbf{x}_{s+m}\right), F_{s+m}\right]-\mu \frac{\partial h_{k}}{\partial p_{1}^{\alpha}} \frac{\partial F_{s+m}}{\partial r^{\alpha}}\right\} .
\end{aligned}
$$

Здесь обозначение скобки Пуассона сохранено для канонических переменных $q, p$; по индексу $\alpha$ подразумевается суммирование от 1 до $n$. Функции $F_{s}\left(\mathbf{r}, x^{s}, t ; \mu\right)$ асимптотически регулярны по $\mu$ в окрестности нуля, т.е.

$$
F_{s}\left(\mathbf{r}, x^{s}, t ; \mu\right) \sim \sum_{k} \mu^{k} F_{s}^{k}\left(\mathbf{r}, x^{s}, t\right),
$$

а ФР нулевого приближения $F_{s}^{0}\left(\mathbf{r}, x^{s}, t\right)$ определяются как локально-равновесные решения стационарной цепочки (1.4), в которые $\mathbf{r}$ и $t$ входят как параметры, причем только через гидродинамические переменные (ГДП). Используя затем симметрию ФР относительно перестановок канонических координат и принцип ослабления корреляций [2], получаем уравнения гидродинамики в виде асимптотических разложений по $\mu$. Такой подход обобщает метод Чепмена-Энскога [6], разработанный для уравнения Больцмана.

\section{2. ЭВОЛЮЦИЯ СРЕДНИХ ВЕЛИЧИН}

Если имеется динамическая величина $s$-кратного типа, симметричная относительно перестановок индексов частищ,

$$
B^{(s)}(x)=\sum_{1 \leqslant i_{1}<\cdots<i_{s} \leqslant N} b_{s}\left(\mathbf{x}_{i_{1}}, \ldots, \mathbf{x}_{i_{s}}\right),
$$

то ее среднее значение в момент времени $t$ в термодинамическом пределе есть [1]

$$
\overline{B^{(s)}}=\frac{1}{s ! \omega^{s}} \int d x^{s} b_{s}\left(x^{s}\right) F_{s} \equiv b_{s}(t) \text {. }
$$

Локальная одночастичная координатная плотность определяется как

$$
\rho(\mathbf{r}, t)=\frac{1}{\omega} \int d \mathbf{p} F_{1}(\mathbf{r}, \mathbf{p}, t) .
$$


Средней локальной плотностью величины $B^{(s)}$ называется величина

$$
\left\langle B_{s}\right\rangle=\frac{1}{\rho \omega^{s} s !} \int d \mathbf{p}_{1} d \mathbf{x}_{2} \ldots d \mathbf{x}_{s} b_{s}\left(x^{s}\right) F_{s} .
$$

Эволюция локальной плотности $\rho(2.3)$ определяется из первого уравнения цепочки (1.5):

$$
\partial_{t} F_{1}+\mu \frac{\partial}{\partial r^{\alpha}}\left(v_{\mathrm{eff}}^{\alpha} F_{1}\right)=\left[H_{1}, F_{1}\right]+\sum_{k=2}^{K} \frac{1}{(k-1) ! \omega^{k-1}} \int d \mathbf{x}_{2} \ldots d \mathbf{x}_{k}\left[h_{k}\left(x^{k}\right), F_{k}\right],
$$

где

$$
v_{\mathrm{eff}}^{\alpha}=\frac{\partial H_{1}}{\partial p_{1}^{\alpha}}+\sum_{k=2}^{K} \frac{1}{(k-1) ! \omega^{k-1}} \frac{1}{F_{1}} \int d \mathbf{x}_{2} \ldots d \mathbf{x}_{k} \frac{\partial h_{k}\left(x^{k}\right)}{\partial p_{1}^{\alpha}} F_{k} .
$$

Эффективная скорость $v_{\text {eff }}$ есть аналог унарной динамической величины, среднее значение которой определяет среднюю локальную скорость частиц системы

$$
u^{\alpha}(\mathbf{r}, t)=\sum_{k=1}^{K} k\left\langle\frac{\partial h_{k}}{\partial p_{1}^{\alpha}}\right\rangle=\frac{1}{\omega \rho} \int d \mathbf{p}_{1} v_{\mathrm{eff}}^{\alpha} F_{1}
$$

Из соотношений $(2.3),(2.5)$ следует, что эволюция локальной плотности определяется уравнением

$$
\partial_{t} \rho=-\mu \frac{\partial}{\partial r^{\alpha}}\left(\rho u^{\alpha}\right)
$$

Обозначим оператор $\mu(\partial / \partial \mathbf{r})$ через $\nabla$. Для эволюции средней локальной плотности (2.4) получаем уравнение

$$
\partial_{t} \rho\left\langle B_{s}\right\rangle=-\nabla_{\alpha} \rho \Pi_{s}^{\alpha}+\rho T_{s},
$$

где введены следующие обозначения:

$$
\begin{aligned}
\Pi_{s}^{\alpha}= & \frac{1}{\rho \omega^{s} s !} \int d \mathbf{p}_{1} d \mathbf{x}_{2} \ldots d \mathbf{x}_{s} b_{s}\left(x^{s}\right)\left\{\frac{\partial H_{s}}{\partial p_{1}^{\alpha}} F_{s}+\sum_{k=2}^{K} \sum_{m=1}^{k-1} \sum_{2 \leqslant i_{2}<\cdots<i_{k-l} \leqslant s} \frac{1}{m ! \omega^{m}} \times\right. \\
& \left.\times \int d \mathbf{x}_{s+1} \ldots d \mathbf{x}_{s+m} \frac{\partial h_{k}\left(x^{k-l}, \mathbf{x}_{s+1}, \ldots, \mathbf{x}_{s+m}\right)}{\partial p_{1}^{\alpha}} F_{s+m}\right\} \\
T_{s}= & \left\langle\left[b_{s}, H_{s}^{\text {eff }}\right]\right\rangle, \quad H_{s}^{\mathrm{eff}}=H_{s}+\sum_{k=2}^{K} \sum_{m=1}^{k-1} \sum_{2 \leqslant i_{2}<\cdots<i_{k-l} \leqslant s} \frac{1}{m ! \omega^{m} F_{s}} \times \\
& \times \int d \mathbf{x}_{s+1} \ldots d \mathbf{x}_{s+m} h_{k}\left(x^{k-l}, \mathbf{x}_{s+1}, \ldots, \mathbf{x}_{s+m}\right) F_{s+m} .
\end{aligned}
$$

Соотношения (2.9) обобщают результаты работ [1], [2] на случай, когда гамильтониан является произвольной функцией координат и импульсов. Таким образом, эволюция локальной плотности $s$-кратной динамической величины определяется дивергенцией потока этой плотности и средним значением скобки Пуассона рассматриваемой динамической величины и эффективного гамильтониана $s$-частичного взаимодействия. Подставляя в $(2.8)$ различные динамические величины $B^{(s)}$, получаем уравнения эволюции 
макроскопических средних величин (ГДП). В гидродинамике используются следующие ГДП, обозначаемые далее $\rho^{\nu}, \nu=1, \ldots, n+2$ :

$$
\rho^{1}=\rho, \quad \rho^{\alpha+1}=\rho\left\langle p^{\alpha}\right\rangle \equiv \rho p^{* \alpha}, \quad \rho^{n+2}=\rho\left\langle\widetilde{H}_{N}\right\rangle \equiv \rho \varepsilon .
$$

Тильда у гамильтониана означает, что он записан в системе отсчета, движущейся со скоростью, отвечающей среднему импульсу p $^{*}$. Согласно функциональной гипотезе нулевое приближение определяется локально-равновесными ФР. Нахождение этих функций для системы взаимодействующих частиц является отдельной сложной задачей, связанной с вычислением конфигурационного интеграла [1], [7], [8], и здесь получение функции $F_{s}^{0}$ не приводится. Локально-равновесные $\Phi$ Р зависят от фазовых переменных через аддитивные интегралы движения, а интенсивные параметры (ИП), сопряженные этим инвариантам, зависят от $\mathbf{r}$ и $t$. Обычно ИП - это плотность, импульс, температура, обозначаемые далее $\pi^{\nu}$, которые затем связываются с ГДП (2.10),

$$
\rho^{\nu}=\left.\rho^{\nu}\right|_{F_{s}^{0}},\left.\quad \rho^{\nu}\right|_{F_{s}^{k}}=0, \quad k \geqslant 1 .
$$

Следует подчеркнуть, что для идеального газа уравнения (2.11) взаимно однозначно определяют ГДП через ИП. В обшем случае требуется невырожденность матрицы

$$
\mathcal{Q}_{\nu}^{\mu}=\frac{\partial \rho^{\mu}}{\partial \pi^{\nu}},
$$

так как уравнения гидродинамики связывают в локально-равновесном приближении временные производные ГДП с градиентами ИП, а для нахождения первой неравновесной поправки к $Ф$ Р надо записать эти уравнения в единых переменных. Например, для идеального релятивистского газа [9] с единичной энергией покоя атома $\left(m c^{2} \equiv 1\right)$ имеем

$$
F_{1}^{0}=\frac{\omega \rho}{Z_{0}} e^{-\beta \widetilde{H}_{0}}
$$

где

$$
Z_{0}=2\left(\frac{2 \pi}{\beta}\right)^{(n-1) / 2} \sqrt{1+s^{2}} K_{(n+1) / 2}(\beta), \quad \widetilde{H}_{0}=\sqrt{\left(1+p^{2}\right)\left(1+s^{2}\right)}-\text { ps. }
$$

Здесь $K_{\nu}(z)$ - модифицированная функция Бесселя второго рода. Параметрами распределения (2.13) являются ИП $\rho, \mathbf{s}, \beta ; \mathbf{s}$ - импульс, который отвечает средней скорости, а $\mathbf{p}^{*}=\langle\mathbf{p}\rangle-$ средний импульс системы,

$$
\begin{gathered}
\mathbf{s}=\frac{\mathbf{u}}{\sqrt{1-u^{2}}}, \quad \mathbf{u}=\left\langle\frac{\mathbf{p}}{\sqrt{1+p^{2}}}\right\rangle, \quad \mathbf{p}^{*}=\gamma \mathbf{s}, \\
\gamma=\frac{K_{(n+3) / 2}(\beta)}{K_{(n+1) / 2}(\beta)}, \quad \varepsilon=\gamma-1-\frac{1}{\beta} .
\end{gathered}
$$

Поскольку $\mathbf{p}^{*}$ зависит от фактора Гиббса $\beta$, возникает проблема разрешимости уравнений гидродинамики первого приближения относительно временных производных ГДП. Для случая (2.14) эта задача разрешима [10], но уже в постньютоновском приближении возможно вырождение матрицы (2.12). В приближении малых слаборелятивистских поправок, когда $\operatorname{det} \mathcal{Q} \neq 0$, можно получить выражения для коэффициентов переноса, если соответствующие классические значения считать известными. 


\section{3. ГИДРОДИНАМИКА ПЕРВОГО ПРИБЛИЖЕНИЯ}

Рассмотрим сначала систему классических частиц единичной массы, которые взаимодействуют через парный потенциал $h_{2}\left(\mathbf{x}_{i}, \mathbf{x}_{j}\right) \equiv \Phi_{i j}=\Phi\left(\left|\mathbf{q}_{i j}\right|\right)$, зависяший только от модуля разности координат. Локально-равновесные $Ф$ Р для такой системы имеют вид [2]

$$
F_{s}^{0}=(\omega \rho)^{s}\left(\frac{\beta}{2 \pi}\right)^{n s / 2} g_{s}\left(q^{s}\right) \prod_{k=1}^{s} e^{-\frac{\beta\left(\mathbf{p}_{k}-\mathbf{u}\right)^{2}}{2}},
$$

где $\rho, \mathbf{u}, \beta$ - функции от $\mathbf{r}$ и $t$, а $g_{s}$ - равновесная координатная $\Phi \mathrm{P}$, зависяшая от $\beta, \rho$ как от параметров; $g_{1} \equiv 1, g_{2}=g\left(\left|\mathbf{q}_{12}\right|\right)$. Согласно (2.11) получаем

$$
\mathbf{p}^{*}=\mathbf{u}, \quad \varepsilon=\frac{1}{2 \beta}(n+U(\rho, \beta)), \quad U(\rho, \beta)=\beta \rho \int d q \Phi(q) g(q) .
$$

Уравнения эволюции ГДП имеют вид [2]

$$
\begin{aligned}
\partial_{t} \rho & =-\nabla_{\alpha} \rho u^{\alpha}, \\
\partial_{t} u^{\alpha} & =-u^{\beta} \nabla_{\beta} u^{\alpha}-\frac{1}{\rho} \nabla_{\beta} \rho P^{\alpha \beta}, \\
\partial_{t} \varepsilon & =-u^{\alpha} \nabla_{\alpha} \varepsilon-\frac{1}{\rho} \nabla_{\alpha} \rho J^{\alpha}-P^{\alpha \beta} \nabla_{\alpha} u^{\beta},
\end{aligned}
$$

где

$$
\begin{aligned}
P^{\alpha \beta} & =\left\langle p^{\alpha} p^{\beta}\right\rangle-u^{\alpha} u^{\beta}-\left\langle q^{\beta} \frac{\partial \Phi(q)}{\partial q^{\alpha}}\right\rangle, \\
J^{\alpha} & =\frac{1}{2}\left\langle p_{1}^{\alpha}(\mathbf{p}-\mathbf{u})^{2}\right\rangle+\left\langle p_{1}^{\alpha} \Phi\right\rangle-u^{\alpha} \varepsilon-\left\langle\left(p_{1}^{\beta}-u^{\beta}\right) q^{\alpha} \frac{\partial \Phi(q)}{\partial q^{\beta}}\right\rangle .
\end{aligned}
$$

В локально-равновесном приближении (3.1) выражения для потоков (3.4) принимают вид

$$
\begin{gathered}
P_{0}^{\alpha \beta}=\frac{1}{\beta} \delta^{\alpha \beta}(1-G(\rho, \beta)), \quad J_{0}^{\alpha}=0, \\
G(\rho, \beta)=\frac{\beta \rho}{2 n} \int d q q \Phi^{\prime}(q) g(q) .
\end{gathered}
$$

Поскольку правые части уравнений (3.3) имеют порядок $O(\mu)$, то

$$
\partial_{t} F_{1}^{0}=F_{1}^{0} \sigma^{\nu} \partial_{t} \pi^{\nu}=O(\mu), \quad \sigma^{\nu}=\frac{\partial \ln F_{1}^{0}}{\partial \pi^{\nu}}=\left(1, \beta \mathbf{w}, \frac{n-\beta w^{2}}{2 \beta}\right), \quad \mathbf{w}=\mathbf{p}-\mathbf{u}
$$

Подставляя разложение (1.6) в уравнение (2.5) и приравнивая в нем члены порядка $O(\mu)$, получаем уравнение для поправки $F_{2}^{1}$ к бинарной локально-равновесной $\Phi \mathrm{P}$,

$$
\sigma^{\nu} \partial_{t} \pi^{\nu}+\left(\sigma^{\nu} v_{\mathrm{eff}}^{\alpha}+\frac{\partial v_{\mathrm{eff}}^{\alpha}}{\partial \pi^{\nu}}\right) \nabla_{\alpha} \pi^{\nu}=\frac{1}{\omega F_{1}^{0}} \int d \mathbf{x}_{2}\left[\Phi_{12}, F_{2}^{1}\right]
$$

Если теперь выразить производные по времени от ИП через градиенты ГДП согласно (3.3) при условии $\operatorname{det} \mathcal{Q} \neq 0$, то функцию $F_{2}^{1}$ можно представить в виде разложения по 
градиентам ИП. В рассматриваемом случае уравнения ГДП (3.3) в терминах ИП имеют вид

$$
\partial_{t} \pi^{\nu}=\Lambda_{\alpha}^{\nu \mu} \nabla^{\alpha} \pi_{\mu}
$$

где коэффициенты $\Lambda_{\alpha}^{\nu \mu}$ зависят от $\pi^{\nu}$ и объединяются в блочную матрицу

$$
\Lambda_{\mathrm{cl}}=\left\|\begin{array}{ccc}
-\mathbf{u} & -\hat{\mathbf{I}} & \mathbf{0} \\
\hat{\mathbf{I}} \frac{1}{\beta}\left(\frac{\partial \rho G}{\partial \rho}-1\right) & \mathbf{u} \hat{\mathbf{I}} & \hat{\mathbf{I}}\left(\frac{1}{\beta^{2}}+\frac{\partial\left(\frac{G}{\beta}\right)}{\partial \beta}\right) \\
\mathbf{0} & \hat{\mathbf{I}} \beta \frac{\rho \frac{\partial U}{\partial \rho}+2(G-1)}{2 \beta \frac{\partial U}{\partial \beta}-U-n} & \mathbf{u}
\end{array}\right\|
$$

где $\mathbf{0}$ - строка длины $n$, а $\hat{\mathbf{I}}$ - единичная $(n \times n)$-матрица. После этого функцию $F_{2}^{1}$ будем искать в виде

$$
F_{2}^{1}=F_{2}^{0} X_{\nu}^{\alpha} \nabla_{\alpha} \pi^{\nu}
$$

Подставляя соотношения (3.8)-(3.10) в уравнение (3.7), получаем уравнение относительно $X_{\nu}^{\alpha}$

$$
\left(\Lambda_{\alpha}^{\nu \mu}+v_{\mathrm{eff}}^{\alpha} \delta^{\nu \mu}\right) \sigma_{\nu}+\frac{\partial v_{\mathrm{eff}}^{\alpha}}{\partial \pi_{\mu}} \equiv Y_{\mu}^{\alpha}=\frac{1}{\omega F_{1}^{0}} \int d \mathbf{x}_{2}\left[\Phi_{12}, F_{2}^{0} X_{\mu}^{\alpha}\right]
$$

где

$$
\begin{gathered}
Y_{1}^{\alpha}=w^{\alpha} \frac{\partial \rho G}{\partial \rho}, \quad Y_{n+2}^{\alpha}=\frac{w^{\alpha}}{2 \beta}\left(n+2-\beta w^{2}+\beta^{2} \frac{\partial}{\partial \beta} \frac{G}{\beta}\right), \\
Y^{\alpha \gamma}=\beta w^{\alpha} w^{\gamma}+\delta^{\alpha \gamma}\left(-1+\frac{1}{2}\left(n-\beta w^{2}\right) \frac{\rho \frac{\partial U}{\partial \rho}+2(G-1)}{2 \beta \frac{\partial U}{\partial \beta}-U-n}\right) .
\end{gathered}
$$

Решение уравнения (3.11) ищется путем разложения неизвестных функций $X_{\mu}^{\alpha}$ в ряды по полиномам Сонина-Лагерра относительно w [6], [9]. Некоторые частные решения этой задачи содержатся в работе [11]. Подстановка (3.10) в (3.4) дает второе приближение для потоков, после чего уравнения гидродинамики (3.3) дополняются членами второго порядка по градиентам ГДП. Ниже эта схема реализуется на примере слаборелятивистских систем с запаздыванием взаимодействия.

\section{4. ПОСТНЬЮТОНОВСКАЯ ГИДРОДИНАМИКА}

Рассмотрим динамическую систему с запаздыванием взаимодействия [3], [12] в постньютоновском приближении, когда в уравнениях движения учитываются поправки порядка $p^{2} / c^{2}$. Примерами таких систем являются приближения Дарвина [13] в электродинамике и Фока [5] в теории тяготения. Гамильтониан, включаюший в себя обе эти модели, имеет вид

$$
\begin{aligned}
H= & \sum_{i} c^{2} \sqrt{1+\frac{p^{2}}{c^{2}}}+\sum_{i<j}\left(\Phi_{i j}+\frac{1}{2 c^{2}} \Phi_{i j}^{\mathrm{R}}\right)+ \\
& +\frac{\kappa}{6 c^{2}} \sum_{i<j<k}\left(\Phi_{i j} \Phi_{i k}+\Phi_{i j} \Phi_{j k}+\Phi_{i k} \Phi_{j k}\right),
\end{aligned}
$$


где $\Phi_{i j}=\Phi\left(\left|\mathbf{q}_{i j}\right|\right) \equiv \Phi_{i j}^{\mathrm{cl}}-$ классический потенциал парного взаимодействия, а функция $\Phi_{i j}^{\mathrm{R}}$ имеет вид

$$
\Phi_{i j}^{\mathrm{R}}=a \Phi_{i j}\left(\mathbf{p}_{i}-\mathbf{p}_{j}\right)^{2}+A_{i j}^{\alpha \beta} p_{i}^{\alpha} p_{j}^{\beta}+b \Phi_{i j}^{2},
$$

где

$$
A_{i j}^{\alpha \beta}=-\Phi_{i j} \delta^{\alpha \beta}+q_{i j} \Phi_{i j}^{\prime} n_{i j}^{\alpha} n_{i j}^{\beta}, \quad n_{i j}^{\alpha}=\frac{q_{i j}^{\alpha}}{q_{i j}} .
$$

При $\kappa=0, a=0, b=0$ из соотношений (4.1), (4.2) получается модель Дарвина, а при $\kappa=1, a=3, b=1$ - модель Фока. По-прежнему полагаем массы всех частиц единичными. Для удобства сравнения с идеальным релятивистским газом и для сохранения правильной асимптотики равновесного распределения кинематические эффекты будем учитывать полностью. Локально-равновесные $Ф$ Р для этой модели были получены в работах [8], [14]. В частности, функция $F_{1}^{0}$ имеет вид

$$
F_{1}^{0}=F_{\mathrm{Yu}}\left[1-\frac{a n}{2 \omega \rho c^{2}}\left((\mathbf{p}-\mathbf{s})^{2}-\frac{n}{\beta}\right) U+o\left(c^{-2}\right)\right] .
$$

Здесь $U$ определена в (3.2), $F_{\mathrm{Yu}}-\Phi$ Р Юттнера [9], определенная в (2.13). При нахождении ГДП в нулевом приближении для $F_{3}^{0}$ используется классическое выражение, поскольку тройные члены в (4.1) уже имеют нужный порядок малости. Тогда для $\mathbf{p}^{*}$ получаем то же выражение, что и в (2.14), а для других ГДП имеем

$$
\begin{aligned}
\mathbf{v}_{\mathrm{eff}} & =\frac{\mathbf{p}}{\sqrt{1+\frac{p^{2}}{c^{2}}}}+\frac{a U}{\beta c^{2}} \mathbf{w}+\frac{2 G-U}{2 \beta c^{2}} \mathbf{s}, \quad \mathbf{u}=\frac{\mathbf{s}}{\sqrt{1+\frac{s^{2}}{c^{2}}}}+\frac{2 G-U}{2 \beta c^{2}} \mathbf{s}, \\
\varepsilon & =c^{2}(\gamma-1)-\frac{1}{\beta}+\frac{U}{\beta}\left(1+\frac{a n}{\beta c^{2}}\right)+\frac{b-a n}{2 \beta^{2} c^{2}} D_{1}+\frac{\kappa}{2 \beta^{2} c^{2}} D_{2} .
\end{aligned}
$$

Здесь $\gamma$ определена в (2.14), $U$ - в (3.2), $G$ - в (3.5), а функции $D_{1}$ и $D_{2}$, учитываюшие характерные для слаборелятивистского приближения поправки к ГДП второго порядка по взаимодействию, имеют вид

$$
D_{1}=\beta^{2} \rho \int \Phi^{2}(q) g(q) d q, \quad D_{2}=\beta^{2} \rho^{2} \int \Phi(q) \Phi\left(\left|\mathbf{q}^{\prime}-\mathbf{q}\right|\right) g_{3}\left(q, q^{\prime}\right) d q d q^{\prime}
$$

Поскольку сами ИП теперь содержатся в определении средней энергии и $\mathbf{u} \neq \mathbf{p}^{*} \neq \mathbf{s}$, то производные ГДП и ИП присутствуют в уравнениях гидродинамики одновременно, т.е.

$$
\begin{gathered}
\partial_{t} \rho=-\nabla_{\alpha} \rho u^{\alpha} \\
\partial_{t} p^{* \alpha}=-u^{\gamma} \nabla_{\gamma} p^{* \alpha}-\frac{1}{\rho} \nabla_{\gamma} \rho P^{\alpha \gamma} \\
\partial_{t} \varepsilon+\frac{1}{\beta\left(c^{2}+s^{2}\right)} s^{\alpha} \partial_{t} s^{\alpha}=-u^{\alpha} \nabla_{\alpha} \varepsilon-\frac{1}{\rho} \nabla_{\alpha} \rho J^{\alpha}+T^{\alpha \gamma} \nabla_{\alpha} s^{\gamma} .
\end{gathered}
$$


В отличие от системы (3.3) в уравнение для энергии входит тензор $T^{\alpha \gamma}$, в общем случае отличный от тензора давления $P^{\alpha \gamma}$. В локально-равновесном приближении величины в правой части (4.6) имеют вид

$$
\begin{gathered}
P_{0}^{\alpha \gamma}=\frac{\delta^{\alpha \gamma}}{\beta}\left(\frac{1}{\sqrt{1+s^{2} / c^{2}}}-G\right)+\frac{\delta^{\alpha \gamma}}{2 \beta^{2} c^{2}}\left[U a(1-n G)+(a n-\kappa) V_{1}+n b V_{2}\right]- \\
-\frac{G}{\beta}\left[\delta^{\alpha \gamma}\left(\frac{n a}{\beta c^{2}}-\frac{s^{2}}{2 c^{2}} \frac{n+3}{n+2}\right)+\frac{s^{\alpha} s^{\gamma}}{c^{2}} \frac{n+1}{n+2}\right]-\frac{B}{2 \beta c^{2}}\left(\delta^{\alpha \gamma} s^{2}+2 s^{\alpha} s^{\gamma}\right), \\
T_{0}^{\alpha \gamma}=-P_{0}^{\alpha \gamma}+\frac{U-G}{2 \beta^{2} c^{2}} \delta^{\alpha \gamma}-B \frac{s^{\alpha} s^{\gamma}}{s^{2}+c^{2}},
\end{gathered}
$$

где введены следуюшие обозначения интегралов:

$$
\begin{gathered}
B=\frac{\beta \rho}{2 n(n+2)} \int q^{2} \Phi^{\prime \prime}(q) g(q) d q, \quad V_{1}=\frac{\beta^{2} \rho}{2 n} \int q\left(\Phi^{2}(q)\right)^{\prime} g(q) d q \\
V_{2}=\frac{\beta^{2} \rho^{2}}{2 n} \int q \Phi^{\prime}(q)\left[\Phi\left(q^{\prime}\right)+\Phi\left(\left|\mathbf{q}-\mathbf{q}^{\prime}\right|\right)\right] g_{3}\left(q, q^{\prime}\right) d q d q^{\prime}
\end{gathered}
$$

Для идеального релятивистского газа и классического газа частиц со взаимодействием, не зависящим от импульсов, поток тепла $\mathbf{J}_{0}$ в (4.6) в линейном приближении по градиентам отсутствует. Учет членов с запаздыванием взаимодействия приводит к качественно новым эффектам,

$$
\begin{aligned}
& \mathbf{J}_{0}=\frac{\mathbf{s}}{4 \beta^{2} c^{2}}\left[(n+2) B+n U-2 G+2 n a D_{1}+V_{3}+(2 G-U)\left(U-2+2 \beta c^{2}(\gamma-1)\right)\right], \\
& V_{3}=\beta^{2} \rho\left[\int \Phi(q)\left(q \Phi^{\prime}(q)-\Phi(q)\right) g(q) d q+\rho \int \Phi(q)\left(q^{\prime} \Phi^{\prime}\left(q^{\prime}\right)-\Phi\left(q^{\prime}\right)\right) g_{3}\left(q, q^{\prime}\right) d q d q^{\prime}\right] .
\end{aligned}
$$

Отметим, что поправки $O\left(c^{-2}\right)$, квадратичные по взаимодействию, появились в потоковых членах (4.7)-(4.9) не только из-за их наличия в гамильтониане $(4.1)$, но и в силу того что скорость теперь зависит от потенциала взаимодействия.

Найденные выражения (4.7)-(4.9) для термодинамических потоков позволяют определить слаборелятивистские поправки к классическим коэффициентам переноса, т.е. построить уравнения ГДП во втором приближении. Для этого в систему (4.6) необходимо подставить $\mathbf{p}^{*}$ из (2.14) и $\varepsilon$ из (4.4), после чего разрешить ее относительно временных производных ИП. Для нахождения правой части в уравнении (3.11) требуется еще определить величины $\sigma^{\nu}$ из (3.6):

$$
\begin{gathered}
\sigma^{1}=1-\frac{\beta n a}{\rho \omega c^{2}}\left(w^{2}-\frac{n}{\beta}\right) \frac{\partial U}{\partial \ln \rho}, \quad \sigma_{2}^{\alpha}=-\frac{s^{\alpha}}{c^{2}+s^{2}}+\beta w^{\alpha}\left(\sqrt{1+\frac{p^{2}}{c^{2}}}+2 U \frac{n a}{\rho \omega c^{2}}\right), \\
\sigma^{3}=\frac{1}{\beta}-(\gamma-1) c^{2}-c^{2} \sqrt{1+\frac{p^{2}}{c^{2}}} \sqrt{1+\frac{s^{2}}{c^{2}}}+\mathbf{p s}-\frac{a U}{\rho \omega c^{2}}\left[w^{2}\left(1+\frac{\partial \ln U}{\partial \ln \beta}\right)-n \frac{\partial \ln U}{\partial \beta}\right], \\
\frac{\partial \mathbf{v}_{\mathrm{eff}}}{\partial \pi^{\nu}}=\frac{2}{c^{2}}\left(a \mathbf{w} \frac{\partial U}{\partial \ln \rho}+\frac{\mathbf{s}}{2} \frac{\partial(G-U)}{\partial \ln \rho}, \frac{\mathbf{I}}{2 c^{2}}(G-(1+2 a) U), a \mathbf{w} \frac{\partial U}{\partial \beta}+\frac{\mathbf{s}}{2} \frac{\partial(G-U)}{\partial \beta}\right) .
\end{gathered}
$$


Представляя далее локально-равновесные ИП в виде

$$
F^{0}=F^{\mathrm{cl}}+\frac{1}{2 c^{2}} F^{\mathrm{R}}+o\left(c^{-2}\right)
$$

(и аналогично потоки первого приближения в уравнениях ГДП (4.6)), получаем, что неизвестные функции $X$ из (3.11), определяющие необратимые потоки, с заданной точностью $o\left(c^{-2}\right)$ определяются из уравнения

$$
\begin{gathered}
\frac{F_{1}^{\mathrm{R}}}{F_{1}^{\mathrm{cl}}} Y^{\mathrm{cl}}+Y^{\mathrm{R}}-\frac{1}{\omega F_{1}^{\mathrm{cl}}} \int d \mathbf{x}_{2}\left(\left[\Phi_{12}^{\mathrm{cl}}, F_{2}^{\mathrm{R}} X^{\mathrm{cl}}\right]+\left[\Phi_{12}^{\mathrm{R}}, F_{2}^{\mathrm{cl}} X^{\mathrm{cl}}\right]\right)= \\
=\frac{1}{\omega F_{1}^{\mathrm{cl}}} \int d \mathbf{x}_{2}\left(\left[\Phi_{12}^{\mathrm{cl}}, F_{2}^{\mathrm{cl}} X^{\mathrm{R}}\right]\right) .
\end{gathered}
$$

Здесь $X^{\mathrm{cl}}$ - решение уравнения (3.11) в классическом случае (без учета запаздывания взаимодействия), $Y^{\mathrm{cl}}$ - выражения из (3.12), а $Y^{\mathrm{R}}$ - аналогичные им выражения, отвечающие потокам (4.7)-(4.9). Последние выражения имеют достаточно громоздкий вид и здесь не приводятся.

Для определения поправок к коэффициентам переноса будут нужны интегралы от них. При этом необходимо учесть представление (3.10) и условия (2.11). Способ определения этих интегралов следующий. Пусть $\varphi_{k}^{\alpha}, \alpha=\left(\alpha_{1}, \ldots, \alpha_{k}\right),-$ решение уравнения вида (3.11)

$$
\frac{1}{\omega F_{1}^{0}} \int d \mathbf{x}_{2}\left[\Phi_{12}, F_{2}^{0} \varphi_{k}^{\alpha}\right]=W_{k}^{\alpha}, \quad W_{k}^{\alpha}=w_{1}^{\alpha_{1}} \ldots w_{k}^{\alpha_{k}} .
$$

Представляя в левой части уравнения (4.10) $Y^{\alpha}=\sum_{k} C_{k}\left[\pi^{\nu}\right] W_{k}^{\alpha}$, находим $X^{\alpha}=$ $\sum_{k} C_{k}\left[\pi^{\nu}\right] \varphi_{k}^{\alpha}$. Этим определяется первая поправка по параметру неоднородности к локально-равновесной бинарной функции (3.10), что позволяет вычислить в этом приближении потоки в (4.6) (обозначим их нижним индексом 1). Например, для нахождения первого приближения к величине $u^{\alpha}=\left\langle v_{\mathrm{eff}}^{\alpha}\right\rangle$ заметим, что при разложении в ряд квадратного корня в (4.4) и последуюшем усреднении с учетом (2.11) отличен от нуля только член $-\left(2 c^{2}\right)^{-1}\left\langle p^{\alpha} p^{2}\right\rangle_{1}^{\text {cl }}$. Потоки тепла и импульса в классическом случае имеют вид

$$
\begin{gathered}
J_{\mathrm{cl}}^{\alpha}=\frac{\lambda_{\mathrm{cl}}}{\beta^{2}} \nabla_{\alpha} \beta=\frac{1}{2}\left\langle p^{\alpha} w^{2}\right\rangle_{1}^{\mathrm{cl}}, \\
P_{\mathrm{cl}}^{\alpha \beta}=-\frac{\eta_{\mathrm{cl}}}{2}\left(\nabla_{\alpha} u^{\beta}+\nabla_{\beta} u^{\alpha}-\frac{2}{n} \delta^{\alpha \beta} \operatorname{div} \mathbf{u}\right) \equiv-\eta_{\mathrm{cl}} \widehat{D}^{\alpha \beta} \mathbf{u}=\left\langle w^{\alpha} w^{\beta}\right\rangle_{1}^{\mathrm{cl}} .
\end{gathered}
$$

Отсюда получаем слаборелятивистскую поправку к локальной скорости

$$
u_{1}^{\alpha}=-\frac{\lambda_{\mathrm{cl}}}{\beta^{2} c^{2}} \nabla_{\alpha} \beta+\frac{\eta_{\mathrm{cl}}}{c^{2}}\left(s^{\alpha}+s^{\beta}\right) \widehat{D}^{\alpha \beta} \mathbf{u} .
$$

Аналогично рассматриваются и другие величины в правой части (4.6), например

$$
J_{1}^{\alpha}=\frac{1}{\beta}\left(\left(1-\frac{s^{2}}{2 c^{2}}\right) \lambda_{\mathrm{cl}}+\frac{1}{c^{2}} \frac{\partial \lambda_{\mathrm{cl}}}{\partial \beta}\right) \nabla_{\alpha} \beta+\frac{1}{c^{2}}\left(\frac{U}{2 \beta} \eta-\frac{\partial \eta}{\partial \beta}\right) s^{\beta} \widehat{D}^{\alpha \beta} \mathbf{u} .
$$


Если система в целом покоится, то из (4.12) следует, что в потоке тепла имеется только поправка к коэффициенту теплопроводности, т.е.

$$
\lambda=\lambda_{\mathrm{cl}}+\frac{1}{c^{2}} \frac{\partial \lambda_{\mathrm{cl}}}{\partial \beta} .
$$

Для тензора давления имеем

$$
\begin{gathered}
P_{1}^{\alpha \gamma}=-\left(\left(1-\frac{s^{2}}{2 c^{2}}\right) \eta_{\mathrm{cl}}+\frac{1}{c^{2}} \frac{\partial \eta_{\mathrm{cl}}}{\partial \beta}\right) \partial_{\mathrm{sym}}^{\alpha \gamma} \mathbf{u}+\frac{s^{\alpha} s^{\beta} \eta_{\mathrm{cl}}}{2 c^{2}} \partial_{\mathrm{sym}}^{\beta \gamma} \mathbf{u}+ \\
+\frac{\lambda_{\mathrm{cl}}}{2 n c^{2}}\left(s^{\alpha} \nabla_{\gamma} \beta+s^{\gamma} \nabla_{\alpha} \beta\right)-\zeta_{\mathrm{R}} \delta^{\alpha \gamma} \operatorname{div} \mathbf{u} \\
T_{1}^{\alpha \gamma}=-P_{1}^{\alpha \gamma},
\end{gathered}
$$

где

$$
\zeta_{\mathrm{R}}=\frac{s^{2}}{n c^{2}}(n B+U-G) \lambda_{\mathrm{cl}}+\frac{G}{\beta c^{2}} \frac{\partial \lambda_{\mathrm{cl}}}{\partial \beta}\left(\beta \frac{\partial \varepsilon}{\partial \beta}\right)^{-1} .
$$

Производная от средней энергии, входящая в выражение для второй вязкости, вычисляется в соответствии с представлением (4.4).

Формулы (4.11)-(4.13) отражают влияние двух слаборелятивистских эффектов. Вопервых, имеет место перенос средней энергии запаздывания взаимодействия при движении системы как целого. Во-вторых, запаздывание учитывается и в самих коэффициентах вязкости и теплопроводности. Появление объемной вязкости $\zeta$ является чисто слаборелятивистским эффектом. В работах [9], [15] для частных случаев уравнения Больцмана показано, что эта вязкость отсутствует как в классическом, так и в ультрарелятивистском случаях.

\section{5. ЗАКЛЮЧЕНИЕ}

В заключение надо сказать об особенностях постньютоновского приближения при выводе уравнений ГДП (4.6), связанных с вырождением преобразования Лежандра [4], [8]. На гидродинамическом уровне система (4.1) имеет три множества особых точек. Первое множество определяет точки вырождения матришы (2.12) $\operatorname{det} \mathcal{Q}_{\nu}^{\mu}=0$, в которых нет взаимно однозначной связи между ИП и ГДП. Это множество описывается условием обрашения в нуль удельной теплоемкости покояшейся системы $\partial \varepsilon / \partial(1 / \beta)=0$. Кроме того, сами уравнения гидродинамики (4.6) должны быть разрешимы относительно временны́х производных ГДП (как функций ИП), т.е. представляться в виде (3.8). Соответствуюшее условие вырождения имеет вид

$$
\frac{\partial \varepsilon}{\partial(1 / \beta)}+\beta \frac{d \gamma}{d \beta} \frac{s^{2}}{c^{2}+s^{2}}=0 .
$$

Наконец, третье множество особых точек определяется условием

$$
\operatorname{det}\left(\frac{\partial u^{\alpha}}{\partial s^{\gamma}}\right)=\left[\frac{1}{\sqrt{1+\frac{s^{2}}{c^{2}}}}+\frac{2 G-U}{2 \beta c^{2}}\right]^{n-1}\left[\frac{1}{\left(1+\frac{s^{2}}{c^{2}}\right)^{3 / 2}}+\frac{2 G-U}{2 \beta c^{2}}\right]=0 .
$$


Оно связано с вырождением гессиана исходной динамической системы (4.1), вследствие чего ИП-импульс $\mathbf{s}$ на этом множестве нельзя выразить через ГДП-скорость $\mathbf{u}$.

Таким образом, строгий подход к выводу уравнений гидродинамики из цепочки уравнений ББГКИ позволяет выявить ряд нетривиальных эффектов, которые могут быть не замечены в рамках использования редуцированных кинетических уравнений, поскольку применение этих уравнений в каждом конкретном случае нуждается в специальном обосновании.

Благодарности. Работа выполнена при финансовой поддержке РФФИ, проект № 01-01-00407.

\section{Список литературы}

[1] Н. Н. Боголюбов. Проблемы динамической теории в статистической физике. М.-Л.: ГИТТЛ, 1946.

[2] Н. Н. Боголюбов. Сб. тр. Ин-та математики АН УССР. 1948. № 10. С. 41.

[3] Р. П. Гайда. ЭЧАЯ. 1982. Т. 13. № 2. С. 427.

[4] Yu. N. Orlov, I. P. Pavlotsky. Physica A. 1988. V. 151. P. 318.

[5] В.А. Фок. Теория пространства, времени и тяготения. М.: Физматлит, 1961.

[6] С. Чепмен, Т. Коулинг. Математическая теория неоднородных газов. М.: ИЛ, 1960.

[7] Yu. N. Orlov, I. P. Pavlotsky. Physica A. 1992. V. 184. P. 558.

[8] Ю.Н. Орлов. Равновесные функции распределения в постгалилеевом приближении. Препринт ИПМ им. М. В. Келдыша РАН № 23. М.: ИПМ им. М. В. Келдыша, 1992.

[9] С. де Гроот, В. ван Леувен, Х. ван Верт. Релятивистская кинетическая теория. М.: Мир, 1983.

[10] Ю.Н. Орлов. Уравнения гидродинамики слаборелятивистской плазмы. Препринт ИПМ им. М.В. Келдыша РАН № 73. М.: ИПМ им. М. В. Келдыша, 1995.

[11] Ю. Н. Орлов. Вывод уравнений гидродинамики. Препринт ИПМ им. М. В. Келдыша АН СССР № 36. М.: ИПМ им. М. В. Келдыша, 1989.

[12] И. П. Павлоцкий. Введение в слаборелятивистскую статистическую механику. М.: Наука, 1987.

[13] C. G. Darwin. Phil. Mag. 1920. V. 39. P. 357.

[14] Ю. Н. Орлов, И. П. Павлоцкий. ДАН СССР. 1989. Т. 304. № 2. С. 329.

[15] Ю. Н. Орлов, И. П. Павлоцкий. Матем. модел. 1989. Т. 1. № 12. С. 31.

Поступила в редакцию 30.VII.2001 г., после доработки 13.VIII.2002 г. 